\title{
Area-preserving curve-shortening flows: from phase separation to image processing
}

\author{
ITALO CAPUZZO DOLCETTA ${ }^{\dagger}$ AND STEFANO FINZI VITA \\ Dipartimento di Matematica, Università di Roma La Sapienza, Piazzale A. Moro 2, 00185 \\ Roma, Italy \\ AND \\ RICCARDO MARCH ${ }^{\S}$ \\ Istituto per le Applicazioni del Calcolo, CNR, Viale del Policlinico 137, 00161 Roma, Italy
}

[Received 20 March 2000 and in revised form 15 March 2002]

\begin{abstract}
Some known models in phase separation theory (Hele-Shaw, nonlocal mean curvature motion) and their approximations by means of Cahn-Hilliard and nonlocal Allen-Cahn equations are proposed as a tool to generate planar curve-shortening flows without shrinking. This procedure can be seen as a level set approach to area-preserving geometric flows in the spirit of Sapiro and Tannenbaum [38], with application to shape recovery. We discuss the theoretical validation of this method and its implementation to problems of shape recovery in Computer Vision. The results of some numerical experiments on image processing are presented.
\end{abstract}

Keywords: Planar curve evolution; phase separation theory; image processing

\section{Introduction}

A commonly adopted mathematical model for a monochromatic plane image is the grey-level representation in which the image is described by a function $g$ defined on a subset $\Omega$ of $\mathbf{R}^{2}$. The real number $g(x, y) \in[0,1]$ is the intensity of grey at the point $(x, y)$ and one of the interesting problems in image processing is to extract from these values the contours of the objects in the image.

On this ground, plane curves deformations have been recently considered in Computer Vision for the purpose of shape recovery and analysis as well as in relation to image segmentation (see, for example, [23-25, 37, 38]). In this context, the curve deformation process should be designed in order to yield both noise suppression in the initial contours and to progressively modify them to a form which is more manageable for pattern recognition and interpretation. A further important feature of the process should be that of providing a representation of the object contours across different scales satisfying the usual properties of multi-scale representation theory such as recursivity, locality, greyscale invariance, and conservation of inclusions (see [3, 25, 29]).

More precisely, if $C(p, t): S^{1} \times[0, T) \rightarrow \mathbf{R}^{2}$ is a representation of a contour curve parametrized by the point $p$ on the curve itself and the real variable $t$ representing scale, the deformation process

\footnotetext{
†Email: capuzzo@mat.uniroma1.it

Corresponding author. Email: finzi@mat.uniroma1.it

${ }^{\S}$ Email: march@iac.rm.cnr.it
} 
can be described by an evolution equation of the form

$$
\frac{\partial C}{\partial t}(p, t)=V(p, t)
$$

where the velocity field $V$ must be chosen in such a way that, as the scale parameter $t$ increases, the initial contour $C(p, 0)$ is deformed according to equation (1.1) into modified contours fulfilling the requirements above.

An important example which has been thoroughly investigated both in mathematical literature $[18,20-22]$ and in Computer Vision [24, 25, 37] is the geometric heat flow:

$$
\frac{\partial C}{\partial t}=\kappa \mathcal{N}
$$

where $\kappa$ and $\mathcal{N}$ are, respectively, the Euclidean curvature and the inward unit normal of $C$. A relevant feature of the flow (1.2) is curve shortening: the evolution defined by equation (1.2) is in fact the fastest way to shrink the length of a curve [19]. The shortening and the related simultaneous smoothing effect induce a desirable noise suppression effect as the scale parameter $t$ increases. On the other hand, a major intrinsic drawback in the application of (1.2) to image processing is the well known fact $[20,22]$ that the area enclosed in a planar embedded curve which evolves according to the geometric heat flow decreases as $t$ increases and that the shape of the object becomes more and more circular and eventually shrinks to a point in finite time. Such a shrinking effect is indeed an undesirable feature in image processing applications: the evolving contour will diverge rapidly from the desired shape causing loss of information.

Two modifications of the geometric heat flow which are simultaneously curve-shortening and area-preserving have been considered by Gage [19], namely

$$
\frac{\partial C}{\partial t}=\left(\kappa-\frac{2 \pi}{\mathcal{L}}\right) \mathcal{N},
$$

and

$$
\frac{\partial C}{\partial t}=\left(\kappa-\frac{\pi \rho}{\mathcal{A}}\right) \mathcal{N}+\alpha \mathcal{T}
$$

In (1.3), where $\mathcal{L}=\mathcal{L}(p, t)$ denotes the length of the curve at scale $t$, the correction term $\frac{2 \pi}{\mathcal{L}} \mathcal{N}$ is the component of the length gradient which lies parallel to the area gradient. In (1.4), $\mathcal{A}=\mathcal{A}(p, t)$ denotes the area of the region enclosed by the curve, $\rho(p, t)=-\langle C(p, t), \mathcal{N}(p, t)\rangle$ is the support function of the curve and $\alpha \mathcal{T}$ is a tangential component which does not change the shape; then, the geometric flow is corrected by a homothety magnifying the plane simultaneously with the evolution. The flow (1.4) has been applied by Sapiro-Tannenbaum [38] to the problem of shape recovery in image processing in order to overcome the above-mentioned undesirable effects of the geometric heat flow.

The discussion above indicates that a relevant task in Computer Vision is that of developing efficient numerical methods for the computation of contours evolving according to lengthshortening and area-preserving flows such as (1.3), (1.4). The direct numerical computation of the previous flows is, however, not an easy task for image processing applications where, in general, 
several object contours have to be simultaneously detected and, therefore, the simultaneous tracking of a large and a priori undetermined number of evolving curves is required.

In this paper, whose content was partially announced in [8], we propose a global approach in order to overcome these computational difficulties which is based on the use of some partial differential equations models in phase separation theory which can be interpreted, perhaps asymptotically, as level set formulations $[12,16,26,33]$ of length-shortening and area-preserving flows of type (1.1). The phase separation models here considered (see Section 3 below) are the Cahn-Hilliard equation and the nonlocal Allen-Cahn equation.

The Cahn-Hilliard equation is

$$
\frac{\partial u}{\partial t}=\frac{1}{\varepsilon} \Delta\left(\omega^{\prime}(u)-\varepsilon^{2} \Delta u\right),
$$

where $\varepsilon>0$ is a small parameter and $\omega(s)$ is a double equal-well potential taking its global minimum value 0 at $s= \pm 1$. The results of Pego [34], Alikakos et al. [1] and Chen [11] show that, as $\varepsilon \rightarrow 0$, the zero level sets of solutions of equation (1.5) tend to solutions of the Hele-Shaw free-boundary problem [10]. It turns out that the evolution of a plane closed curve according to the Hele-Shaw model is a curve-shortening motion which does not change the area of the region enclosed by the curve (see, for example, [34]). The corresponding flow can therefore be interpreted as a curve deformation of type (1.1) with a velocity field $V$ implicitly defined by the solution of the Hele-Shaw problem (see Section 2).

The nonlocal Allen-Cahn equation is

$$
\frac{\partial u}{\partial t}=\Delta u-\frac{1}{\varepsilon^{2}} \omega^{\prime}(u)+\frac{1}{\varepsilon^{2}|\Omega|} \int_{\Omega} \omega^{\prime}(u) \mathrm{d} x,
$$

where $\varepsilon>0$ and $\omega(s)$ have the same meaning as in (1.5). By the works of Rubinstein and Sternberg [35] and Bronsard and Stoth [6] it is known that, as $\varepsilon \rightarrow 0$, the zero level sets of solutions of equation (1.6) evolve according to the general flow (1.1) with normal velocity given by

$$
V=\kappa-\frac{1}{|\Gamma|} \int_{\Gamma} \kappa \mathrm{d} s,
$$

| $\Gamma \mid$ denoting the total length of the set of curves $\Gamma$. It is worth to observe that, for a single planar curve, the motion defined by (1.7) reduces to the one given by (1.3).

We then propose the use of equations (1.5) and (1.6) as computational tools for the recovery of shapes from images for the following reasons. First, the nonlocal flows obtained as asymptotic limits from such equations (Hele-Shaw and (1.7), respectively) should indeed regularize the contours of an image without pushing the evolving curves far away from the desired object boundaries, because such flows are both curve-shortening and area-preserving. Moreover, the solution of the Cahn-Hilliard and of the nonlocal Allen-Cahn equations yields automatically also a solution of the above-mentioned problem of detection and simultaneous tracking of an unknown number of evolving curves. A further advantage of the proposed approach lies in the possibility (see Sections 4 and 5) to adapt efficiently to the Computer Vision problem the sophisticated numerical analysis machinery developed for phase separation equations, see $[4,14,30]$. 
Another relevant feature of the limit flows for the application to shape recovery is the global character of the area conservation property: small 'blobs' due to the presence of noise in the image do not persist during the evolution because of the area conservation. On the contrary, a coarsening process associated to the phase separation equations forces, in general, finer structures to disappear as time evolves [35]. Consequently, the related models for shape recovery and analysis show an interesting noise suppression property which consists in a shrinking effect on small blobs. The results of numerical experiments on both synthetic and real images reported in the paper seem to validate this approach.

To conclude this introduction, let us mention that a computational approach which is conceptually similar to the one proposed in this paper but restricted to the curve-shortening flow (1.2) has been previously suggested by Nochetto and Verdi [31]. They consider the Allen-Cahn equation of the phase transition theory [2]

$$
\frac{\partial u}{\partial t}=\Delta u-\frac{1}{\varepsilon^{2}} \omega^{\prime}(u)
$$

where $\varepsilon>0$ and $\omega$ are as in (1.5) and (1.6). Asymptotic results (see $[5,13,15])$ show that, as $\varepsilon \rightarrow 0$, $u$ tends to either +1 or -1 , in two regions separated by a sharp interface which moves by its mean curvature. Therefore, as $\varepsilon \rightarrow 0$, the interface is the zero level set of the solution $u$ and it consists of a set of curves which evolves according to equation (1.2). The numerical solution of the Allen-Cahn equation was then used as a tool for the effective computation of the geometric heat flow which handles changes of topology during the evolution $[30,31]$.

\section{Area-preserving geometric flows}

In this section we review some known properties of area-preserving curve-shortening flows and of phase separation models which yield such flows for the interface and we point out their relevance for shape recovery. Let us observe preliminarily that the geometric heat flow (1.2) is the gradient flow of the length functional on the space of smooth plane curves endowed with the Euclidean metric. If $C(p, t)$ is a one-parameter family of curves with length $\mathcal{L}(t)$, then (see [19])

$$
\frac{\mathrm{d} \mathcal{L}}{\mathrm{d} t}=-\int\left\langle\frac{\partial C}{\partial t}, \kappa \mathcal{N}\right\rangle \mathrm{d} s,
$$

where $s$ denotes the arclength. So the geometric heat flow is the fastest way to shrink the length of a curve. To keep the area enclosed by the curve constant while decreasing the length, Gage proposed to subtract the component of the length gradient which lies parallel to the area gradient. Since the first variation of the area is [19]

$$
\frac{\mathrm{d} \mathcal{A}}{\mathrm{d} t}=-\int\left\langle\frac{\partial C}{\partial t}, \mathcal{N}\right\rangle \mathrm{d} s,
$$

and, for simple closed curves,

$$
\int\langle\kappa \mathcal{N}, \mathcal{N}\rangle \mathrm{d} s=\int \kappa \mathrm{d} s=2 \pi
$$


the geometric evolution equation

$$
\frac{\partial C}{\partial t}=\left(\kappa-\frac{2 \pi}{\mathcal{L}}\right) \mathcal{N}
$$

was proposed as the gradient flow of the length functional along curves which enclose a fixed area.

An alternative method suggested in [19] is to use the geometric heat flow (1.2) while simultaneously magnifying the plane by a homothety which keeps constant the enclosed area. The corresponding evolution equation becomes

$$
\frac{\partial C}{\partial t}=\left(\kappa-\frac{\pi \rho}{\mathcal{A}}\right) \mathcal{N}+\alpha \mathcal{T},
$$

where $\rho=-\langle C, \mathcal{N}\rangle$ is the support function of the curve and $\alpha \mathcal{T}$ is a tangential component which does not affect the shape. The following theorem about the evolutions (2.1) and (2.2) has been proved in [19].

THEOREM 2.1 A smooth convex curve in the plane which evolves according to either (2.1) or (2.2) remains convex and converges to a circle in the $C^{\infty}$ metric.

REMARK 2.2 Since the flow defined by (2.2) and the geometric heat flow are related by dilations, the two flows share the same scale-space properties. The flow (2.2) has been proposed by Sapiro and Tannenbaum for shape recovery in order to overcome the undesirable shrinking effect of the geometric heat flow (see [38]). Conversely, the nonlocal flow (2.1) lacks some important properties like scale invariance and conservation of inclusions; observe in this respect that if two curves are tangential at some point and the inner curve is shorter than the outer one, then at some later time the regions bounded by the evolved curves will certainly overlap (see [19]). Moreover, a nonconvex curve evolving through (2.1) can develop singularities in finite time (see [35]). However, the collision and the subsequent fusion of different portions of a curve during the evolution can be interpreted as the removal of features in the multi-scale representation.

The generalization of (2.1) to the case of many different fronts evolving at the same time has of course an interest in phase separation theory; it corresponds to take in the equation the velocity field (1.7), that is

$$
\frac{\partial C}{\partial t}=\left(\kappa-\frac{1}{|\Gamma|} \int_{\Gamma} \kappa \mathrm{d} s\right) \mathcal{N},
$$

whose solution defines the so-called nonlocal mean curvature flow.

A conceptually completely different way to generate flows having similar geometric properties is, not surprisingly, via the motion of interfaces in phase separation models where conservation of mass enters as a constitutive law.

Consider for example the Hele-Shaw model: let $\Omega$ be a bounded and simply connected domain in $\mathbf{R}^{2}, \Gamma_{*}$ be a smooth closed curve in $\Omega$ and consider the free-boundary problem of finding a 
function $\mu(x, t), t \in[0, T]$, and a free boundary $\Gamma=\cup_{0 \leqslant t \leqslant T}\left(\Gamma_{t} \times\{t\}\right)$ satisfying

$$
\begin{cases}\Delta \mu(\cdot, t)=0 & \text { in } \Omega \backslash \Gamma_{t}, t \in[0, T], \\ \frac{\partial \mu}{\partial n}=0 & \text { on } \partial \Omega \times[0, T], \\ \mu=\lambda \kappa & \text { on } \Gamma_{t}, t \in[0, T], \\ \frac{1}{2}\left[\frac{\partial \mu}{\partial n}\right]_{\Gamma_{t}}=V & \text { on } \Gamma_{t}, t \in[0, T], \\ \Gamma_{0}=\Gamma_{*} & \text { on }\{t=0\},\end{cases}
$$

where $\lambda$ is a positive constant related to the surface tension, $n$ is the unit outward normal to $\partial \Omega$ or to $\Gamma_{t}, \kappa$ and $V$ are, respectively, the curvature and the normal velocity of $\Gamma_{t}$ (with the sign convention that the curvature of a circle and the normal velocity of a shrinking curve are positive),

$$
\left[\frac{\partial \mu}{\partial n}\right]_{\Gamma_{t}}=\frac{\partial \mu^{-}}{\partial n}-\frac{\partial \mu^{+}}{\partial n}
$$

and $\mu^{+}$and $\mu^{-}$are the restrictions of $\mu$ on $\Omega_{t}^{+}$and $\Omega_{t}^{-}$(respectively, the exterior and interior of $\Gamma_{t}$ in $\Omega$ ).

Chen [10] established the local existence of a solution of the Hele-Shaw problem for an arbitrary smooth initial curve, and global existence of a solution when the initial curve is close to a circle.

If we denote by $\mathcal{A}(t)$ and $\mathcal{L}(t)$ the area of $\Omega_{t}^{-}$and the length of $\Gamma_{t}$, respectively (and we use the same notations also for the interface of the flow induced by (2.3)), the following features are relevant for the application of the two flows to the problem of shape recovery [10,34,35].

PROPOSITION 2.3 The following properties hold for the solutions of (2.3) and of (2.4):

$$
\frac{\mathrm{d} \mathcal{A}}{\mathrm{d} t}=0, \quad \frac{\mathrm{d} \mathcal{L}}{\mathrm{d} t} \leqslant 0 .
$$

Therefore, both the Hele-Shaw flow and the nonlocal mean curvature flow preserve the area of the region enclosed by $\Gamma_{t}$ while decreasing its length. In the general case, which occurs in image processing, different fronts evolve at different speeds keeping the total area fixed. A gradual coarsening process takes place since the only possible equilibrium state should be that of one or more discs of the same size.

Let us now consider the theoretical effects of these geometric flows on the evolution of a set of approximate contours $\Gamma_{*}$ extracted from a given image (we will discuss in Section 4 possible ways to do that). Such a set, under suitable hypotheses, can be considered as the union of several nonintersecting closed curves. The curve evolution generated by the Hele-Shaw (or the nonlocal mean curvature) flow starting from $\Gamma_{*}$ should then regularize the contours without pushing the evolving curves far away from the true contours because the flow is both curve-shortening and area-preserving.

One could wonder if small 'blobs' due to the presence of noise in the image data could persist during the evolution because of area conservation. This event is not possible, since a noise suppression property due to a shrinking effect on small blobs takes place. More precisely, for the Hele-Shaw flow we have the following proposition. 
Proposition 2.4 Suppose that one of the initial curves in $\Gamma_{0}$ for the Hele-Shaw flow is a circle with radius $r$. Then, if $r$ is small enough, the circle shrinks.

Proof. From (2.4), $\mu(x, 0)$ is harmonic in $\Omega \backslash \Gamma_{0}$ and constant on the circle, then it is constant inside the circle. Hence on the boundary of the circle we have

$$
\mu(x, 0)=\frac{\lambda}{r}, \quad V=-\frac{1}{2} \frac{\partial \mu^{+}}{\partial n} \quad \text { at } \quad t=0 .
$$

By the maximum principle for harmonic functions, $\mu(x, 0)$ takes then its maximum value on $\Gamma_{0} \cup$ $\partial \Omega$. For small enough $r$, we can assume that $\mu(x, 0)$ takes the maximum value $\lambda / r$ on the boundary of the circle, then from the maximum principle it follows easily that on the boundary of the circle we have

$$
\frac{\partial \mu^{+}}{\partial n}<0
$$

Hence (2.6) implies $V>0$, so that the circle shrinks.

A similar property holds for the nonlocal curvature flow: this is a well known phenomenon in phase transition theory related to coarsening processes in which finer structures disappear as time evolves.

Summing up, the propagation law for free boundaries that arises in the two-phase separation models considered here exhibits the following properties which are useful for shape recovery:

- curve smoothing and multi-scale representation by means of curve-shortening: suppression of noise and removal of small-scale features as time (i.e. scale) increases;

- preservation of area: the main evolving contours do not shrink, then they diverge less rapidly from the desired shape than in the geometric heat flow (1.2);

- simultaneous evolution of all the contours in the image with suppression of small contours due to the presence of noise.

The set $\Gamma_{*}$ contains a noisy version of the contours in the image plus a large number of small blobs caused by noise. Because of the curve-shortening effect, after a small time the curvature of $\Gamma_{t}$ is largest at the boundaries of the blobs. Then the coarsening process starts and the small blobs due to the presence of noise disappear. Very small particulars in the image are treated as noise, here as in any other approach, the goal being the detection of the essential contours. The surviving curves can be considered as the boundaries of the objects of interest. The curve-shortening effect removes noise from those remaining boundaries and the further evolution yields a simultaneous multi-scale representation for all the shapes in the image. As usual in shape recovery by means of curve evolution, the process has to be stopped after a suitable time in order to avoid losing information as $t$ becomes larger and larger.

\section{Shape recovery by phase separation equations}

In this section we shortly review the approximation of the Hele-Shaw model (2.4) by means of the Cahn-Hilliard equation $(1.5)[1,11,34]$ and that of the nonlocal curvature model (2.3) by means of the nonlocal Allen-Cahn equation (1.6) $[6,35]$. 
It is well known from phase separation theory, that the curve evolution law obtained as the limit of a rescaled partial differential equation depends on how the time variable in the equation is rescaled. In the previous section we focused our attention on laws for curve evolution with specific properties that are relevant for image processing (mainly, the conservation of the area enclosed by the fronts), such as the Hele-Shaw and the nonlocal mean curvature flows. From the implementation point of view in image processing however, the direct use of these models is not easy and we found much more convenient their approximation via the Cahn-Hilliard and nonlocal Allen-Cahn equations, used with the corresponding time rescaling (1.5) and (1.6). We then discuss how an algorithm for shape recovery, based on these results, can be designed.

\subsection{Asymptotic behaviour of the models}

In [34] Pego showed by using formal matched asymptotic expansions that level sets of solutions to the Cahn-Hilliard equation tend to solutions of the Hele-Shaw problem as $\varepsilon \rightarrow 0$. Alikakos et al. [1] then rigorously proved Pego's result under the assumption that classical solutions of the Hele-Shaw problem exist and, more recently, Chen [11] removed such a regularity hypothesis.

We rewrite the Cahn-Hilliard equation (1.5) as the system

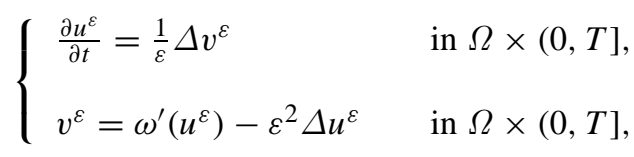

supplemented with initial and boundary conditions

$$
\begin{cases}u^{\varepsilon}(x, 0)=u_{0}^{\varepsilon}(x) & \text { in } \Omega, \\ \frac{\partial u^{\varepsilon}}{\partial n}(x, t)=\frac{\partial v^{\varepsilon}}{\partial n}(x, t)=0 & \text { on } \partial \Omega \times(0, T] .\end{cases}
$$

The property of mass conservation holds as a direct consequence of (3.1) and (3.2):

$$
\int_{\Omega} u^{\varepsilon}(x, t) \mathrm{d} x=\int_{\Omega} u_{0}^{\varepsilon}(x) \mathrm{d} x \quad \forall t \in(0, T] .
$$

Let $\Gamma_{*}$ be a smooth closed curve in $\Omega$ and assume that the Hele-Shaw problem (2.4) starting from $\Gamma_{*}$ has a smooth solution $(\mu, \Gamma)$, with

$$
\Gamma:=\bigcup_{0 \leqslant t \leqslant T}\left(\Gamma_{t} \times\{t\}\right)
$$

in a time interval $[0, T]$ with $\Gamma_{t} \subset \Omega$ for all $t \in[0, T]$. The following theorem has been proved in [1].

THEOREM 3.1 There exists a family of smooth initial data $\left\{u_{0}^{\varepsilon}(x)\right\}_{0<\varepsilon \leqslant 1}$ which are uniformly bounded in $\varepsilon \in(0,1]$ such that if $u^{\varepsilon}$ are the corresponding solutions of (3.1)-(3.2) then

$$
\begin{gathered}
\lim _{\varepsilon \rightarrow 0} u^{\varepsilon}(x, t)=\left\{\begin{array}{ll}
-1 & \text { if }(x, t) \in Q_{0}^{-}, \\
+1 & \text { if }(x, t) \in Q_{0}^{+},
\end{array} \quad\right. \text { uniformly on compact subsets, } \\
\lim _{\varepsilon \rightarrow 0}\left(\varepsilon \Delta u^{\varepsilon}-\frac{1}{\varepsilon} \omega^{\prime}\left(u^{\varepsilon}\right)\right)(x, t)=\mu(x, t) \quad \text { uniformly on } \bar{\Omega} \times(0, T],
\end{gathered}
$$


where $Q_{0}^{+}$and $Q_{0}^{-}$denote, respectively, the exterior (in $\Omega \times(0, T]$ ) and the interior of $\Gamma,(\mu, \Gamma)$ being a solution of (2.4).

If $u^{\varepsilon}$ is a solution to (3.1)-(3.2) and we consider the zero level set

$$
\Gamma^{\varepsilon}:=\left\{(x, t) \in \Omega \times[0, T]: u^{\varepsilon}(x, t)=0\right\}=\bigcup_{0 \leqslant t \leqslant T}\left(\Gamma_{t}^{\varepsilon} \times\{t\}\right),
$$

then $\Gamma$ is the limit front of $\Gamma^{\varepsilon}$ as $\varepsilon \rightarrow 0$, in the sense made precise by the previous theorem.

The above-mentioned result shows that the zero level set of the solution to the Cahn-Hilliard equation approximates as $\varepsilon \rightarrow 0$ a free boundary that solves the Hele-Shaw problem. In particular, the mass conservation law (3.3), taking (3.4) into account, corresponds to the approximate conservation of the area of the region enclosed by the zero level set $\Gamma^{\varepsilon}$, which becomes exact as $\varepsilon \rightarrow 0$.

In a similar way, let us consider now the nonlocal Allen-Cahn problem

$$
\begin{cases}\frac{\partial u^{\varepsilon}}{\partial t}=\Delta u^{\varepsilon}-\frac{1}{\varepsilon^{2}} \omega^{\prime}\left(u^{\varepsilon}\right)+\frac{1}{\varepsilon^{2}|\Omega|} \int_{\Omega} \omega^{\prime}\left(u^{\varepsilon}\right) \mathrm{d} x & \text { in } \Omega \times(0, T], \\ u^{\varepsilon}(x, 0)=u_{0}^{\varepsilon}(x) & \text { in } \Omega, \\ \frac{\partial u^{\varepsilon}}{\partial n}(x, t)=0 & \text { on } \partial \Omega \times(0, T],\end{cases}
$$

where the nonlocal term in the equation plays the role of a Lagrangean multiplier in order to preserve mass. In [35] the method of matched asymptotic expansions has been used to show that as $\varepsilon \rightarrow 0$ the motion of the zero level sets of the solutions of (3.5) describes a coarsening process, with velocity given by equation (1.7), which preserves the total area inside the fronts. A rigorous proof of this convergence has been given, at least for the case of radially symmetric solutions of (3.5) in a bounded spherically symmetric domain $\Omega$, by Bronsard and Stoth [6].

REMARK 3.2 For a more detailed comparison between the flows induced by (3.1)-(3.2) and (3.5) and between their asymptotic models, see [6,35]; the main difference perhaps lies in the case of a multifront evolution. If we consider for example an initial set made of two or more non-intersecting discs, their behaviour is not the same. In the nonlocal mean curvature flow the discs keep their form as time evolves, while shrinking in the increasing order of their size. In the Hele-Shaw flow, on the contrary, the discs distort immediately, and the effects of even very small discs close to extinction is still considerable on the whole flow. This appears as a possible breakdown in the convergence of the Cahn-Hilliard model towards the Hele-Shaw problem in the presence of asymptotically small discs, even if the essential qualitative properties are preserved.

REMARK 3.3 It should be remarked (see [35]), that both the Cahn-Hilliard and the nonlocal Allen-Cahn models can be interpreted as particular cases of the generalized viscous Cahn-Hilliard equation

$$
\alpha \frac{\partial u}{\partial t}=\Delta\left[\omega^{\prime}(u)-\beta \Delta u+v \frac{\partial u}{\partial t}\right],
$$

introduced by Novick-Cohen [32] to take care of viscous effects in phase separation models: $v \rightarrow 0$ yields the Cahn-Hilliard equation, while $\alpha \rightarrow 0$ gives the nonlocal Allen-Cahn equation. In principle, other choices of $\alpha$ and $v$ could yield interesting models for shape recovery. 


\subsection{Application to shape recovery}

The convergence results reviewed in the previous section, together with the properties of the limit models already discussed in Section 2, suggested to us the use of the systems (3.1)-(3.2) or (3.5) to build a numerical algorithm for shape recovery in image segmentation applications: the zero level set of their solutions after a short time can be used to recover the boundaries of the objects of interest in an image.

The double-well potential $\omega$ can be replaced for practical purposes by a double-obstacle potential such as

$$
\psi(s):= \begin{cases}1-s^{2} & \text { if } s \in[-1,1], \\ +\infty & \text { if } s \notin[-1,1] .\end{cases}
$$

With such a choice (see for example [4] for the Cahn-Hilliard model and [30] for the Allen-Cahn model), the essential properties of the flows are preserved, while the solutions (which are now solutions of a double-obstacle variational inequality) are forced to remain in the interval $[-1,1]$ for all times; more than that, they differ from the extrema \pm 1 only on a narrow transition region of size proportional to $\varepsilon$, so that the use of a dynamic mesh algorithm becomes possible (as in [30]).

We remark that this approach can be still considered as a level set method, since an arbitrary and a priori unknown number of contours corresponding to several objects can be simultaneously tracked and recovered from an image, even though, of course, we are not interested in changes of topology during the evolution of the curves that are object boundaries. Furthermore, the coarsening effect, which takes place in these models, makes the small contours due to the presence of noise vanish during the evolution.

Then, an algorithm for shape recovery based on the previous phase separation models should contain the following steps:

- choose an initial set of contours by means of a suitable pre-processing of the image (see next secion);

- compute initial data for the phase separation equation from the initial set of curves;

- compute a solution of the equation (several numerical methods are well known for these models in phase transition theory);

- stop the computation at a suitable time $t$ in order to save the information contained in the evolving contour (see the comment below);

- plot the zero level set of the solution at different times obtaining a multi-scale representation of the shapes contained in the original image.

Here, as in related approaches, the choice of the stopping time is a delicate task and essentially an open problem. One way to implement the stopping criterion is to modify the evolution equation by introducing an interaction with the image intensity function $g$ in order to moderate the geometric diffusion effect when the evolving curve drifts far away from the original shape. For instance, this interaction can be introduced in the Cahn-Hilliard equation by simply replacing the first equation in (3.1) with

$$
\frac{\partial u^{\varepsilon}}{\partial t}=\frac{1}{\varepsilon} \nabla \cdot\left(b(x) \nabla v^{\varepsilon}\right) \quad \text { in } \Omega \times(0, T],
$$


where a suitable form for $b$ can be the following:

$$
b(x)=\frac{1}{1+\eta\left|\nabla G_{\sigma} * g\right|^{2}} .
$$

Here, $\eta$ is a positive parameter and $G_{\sigma}$ is the standard 2D Gaussian function with extent $\sigma$, while * denotes the convolution product. In such a way the mass conservation property still holds while the evolution is slowed down near the 'edges' of $g$, that is for large values of its gradient (for a similar approach in the mean curvature motion case see [9]). The study of such modified models will be the object of a forthcoming paper [17]. A different approach based on optimal control ideas has been proposed in [7].

A final remark is that most of the results discussed in this paper generalize to any space dimension, so that the models of shape recovery based on the analogy with phase transitions can also be applied, in principle, to recover surfaces which are the boundaries of objects in three-dimensional images.

\section{Numerical methods and computational aspects}

In this section we discuss how efficient numerical methods developed in the context of phase transition theory can be adapted to the problem of shape recovery, with several convergence results and error estimates already proved for the discrete solutions. Moreover, here and in the next section we study the application of those models to the analysis of test images, discussing how some known algorithms from the literature can be tailored to fit in our framework.

The first and common issue in such applications to shape recovery is the extraction of a suitable initial set of contours from the given image datum $g$ to be processed. Such a set, here already denoted by $\Gamma_{*}$, can be considered in the simplest case as the union of several nonintersecting closed curves. A possible choice, suggested by Shah [39], is to take

$$
\Gamma_{*}=\left\{x \in \Omega:\left(\Delta G_{\sigma} * g\right)(x)=0, \nabla\left(\Delta G_{\sigma} * g\right)(x) \neq 0\right\} .
$$

The computation of the zero-crossings of the image convolved with the Laplacian of a Gaussian function was originally proposed by Marr and Hildreth [28] as a theory of edge detection. It may be shown [40] that, if $\nabla\left(\Delta G_{\sigma} * g\right)(x) \neq 0$ at any point $x$ where $\left(\Delta G_{\sigma} * g\right)(x)=0$, then the zerocrossing contours are closed curves or curves that terminate at the boundary of the image. For small $\sigma$ the initial set $\Gamma_{*}$ turns out to be close to the 'true' contours of the image, even if still noisy [28].

\subsection{The Cahn-Hilliard approach}

We adopted the splitting method proposed in [14]: the fourth-order Cahn-Hilliard equation (1.5) is first rewritten as the system (3.1)-(3.2) of second-order equations in the two variables $\left(u^{\varepsilon}, v^{\varepsilon}\right)$, and then semidiscretized using continuous piecewise linear finite elements. If $\mathcal{T}_{h}$ denotes a quasiuniform triangulation of $\Omega$ of 'size' $h$, the finite element space will be

$$
V_{h}=\left\{\phi \in C^{0}(\bar{\Omega}):\left.\phi\right|_{\tau} \in \mathbf{P}_{1}, \forall \tau \in \mathcal{T}_{h}\right\},
$$


where $\mathbf{P}_{1}$ is the set of first-order polynomials, and the splitting algorithm becomes (for simplicity we have dropped the dependence on $\varepsilon$ )

$$
\begin{aligned}
& \text { find }\left\{u_{h}, v_{h}\right\}:[0, T] \rightarrow V_{h} \times V_{h} \text { such that } \\
& \qquad \begin{cases}\left(\frac{\partial u_{h}}{\partial t}, \phi\right)^{h}+\frac{1}{\varepsilon}\left(\nabla v_{h}, \nabla \phi\right)=0 & \forall \phi \in V_{h}, \\
\varepsilon^{2}\left(\nabla u_{h}, \nabla \phi\right)+\left(\omega^{\prime}\left(u_{h}\right)-v_{h}, \phi\right)^{h}=0 & \forall \phi \in V_{h}, \\
u^{h}(0)=u_{0}^{h}, & \end{cases}
\end{aligned}
$$

where $u_{0}^{h}$ is a suitable approximation of the initial datum, and (., . $)^{h}$ denotes the approximated $L^{2}$ inner product, given by

$$
(z, w)^{h}=\sum_{\tau \in \mathcal{T}_{h}} \int_{\tau} \Pi_{\tau}^{h}(z(x) w(x)) \mathrm{d} x, \quad \forall z, w \in C^{0}(\bar{\Omega}),
$$

$\Pi_{\tau}^{h}$ being the linear interpolation operator on $\tau$. Let $\left\{\phi_{i}\right\}_{i}$ denote the set of basis functions for $V_{h}$, and $M$, respectively $K$, the (lumped) mass and stiffness matrices of the finite element method; then, if we set

$$
u_{h}(t)=\sum_{i} U_{i}(t) \phi_{i}
$$

the system (4.2) reduces to the following first-order nonlinear system of ordinary differential equations:

$$
\left\{\begin{array}{l}
M \frac{\mathrm{d} U(t)}{\mathrm{d} t}+\varepsilon\left(K M^{-1} K\right) U(t)+\frac{1}{\varepsilon}\left(K M^{-1}\right) \Phi(U(t))=0, \\
U(0)=u_{0}^{h},
\end{array}\right.
$$

where $\Phi(U)$ represents the nonlinear term coming from (4.2).

In [14] this approach is proved to yield second-order optimal error bounds for $\left(u-u_{h}\right)$ in the $L^{\infty}\left(0, T ; L^{2}(\Omega)\right)$ norm, provided the term $u_{0}^{h}$ is suitably chosen, keeping at the same time all the relevant properties of the continuous model (such as conservation of mass).

Concerning the time discretization of (4.3), explicit algorithms are useless in practice (a straightforward stability condition would require a very small time step $\Delta t$, according to the condition number of the matrix $K M^{-1} K$ ). The use of a Crank-Nicholson scheme coupled with the linearization of the term $\Phi(U)$ gave satisfactory results in the tests. Instability effects are still present but they do not affect heavily the scheme; actually, only a short time evolution needs to be considered for our purposes (see the discussion in Section 3.2).

Of course, a fully implicit scheme could be used as well, yielding in principle unconditional stability. We tested for example the scheme proposed in [4] for the Cahn-Hilliard model with nonsmooth free energy, that is with the double-obstacle potential (3.6); in such a way the problem can be written as a variational inequality, and the discretization error estimate for the scaling corresponding to (3.1)-(3.2) reads as

$$
\left\|u-u_{h}\right\|_{L^{\infty}\left(0, T ;\left(H^{1}(\Omega)\right)^{\prime}\right)}^{2}+\left\|u-u_{h}\right\|_{L^{2}\left(0, T ; H^{1}(\Omega)\right)}^{2} \leqslant c\left(\frac{h^{4}}{\Delta t}+h^{2}+\Delta t\right),
$$


where $(V)^{\prime}$ denotes the usual dual space of a space $V$. However, in order to prove this estimate, as well as the convergence of the iterative scheme needed at any step to advance in time, a strong bound on $\Delta t$ is required with respect to $\varepsilon$ (that is $\Delta t<4 \varepsilon^{2}$ ); since a small $\varepsilon$ is necessary for a sharp detection of the contours, and the optimal order of convergence holds when $\Delta t$ is of the order of $h^{2}$, the advantages of the fully implicit scheme are in this case partially lost.

\subsection{The nonlocal Allen-Cahn approach}

For the numerical treatment of the nonlocal Allen-Cahn model (3.5) we preferred to adopt the finite element approach of [30]. Making use of the obstacle potential (3.6), the problem is then reduced to a variational inequality and hence to a complementarity system whose resolution leads to the following explicit iterative scheme: for any $n=0,1, \ldots$ we look for a pair $\left(U^{n+1}, \Lambda^{n+1}\right)$ such that

$$
\begin{aligned}
& \left\{\begin{array}{l}
U^{n+1 / 2}=M^{-1}\left(\left(M-\Delta t K+\frac{\Delta t}{\varepsilon^{2}} M\right) U^{n}+\Delta t \frac{\pi}{4 \varepsilon} M \Lambda^{n+1}\right), \\
U^{n+1}=\mathcal{P} U^{n+1 / 2},
\end{array}\right. \\
& \int_{\Omega} U^{n+1} \mathrm{~d} x=m_{0},
\end{aligned}
$$

where $U^{n}$ denotes the vector of the nodal values at time $t_{n}=n \Delta t, \mathcal{P}$ is the componentwise projection on the interval $[-1,1]$, while $\Lambda^{n+1} \in \mathbf{R}$ is a dynamic Lagrange multiplier needed to fulfil the mass constraint (4.5), with the constant $m_{0}$ deduced from the initial data $U^{0}$ (as before, $M$ and $K$ are the finite element mass and stiffness matrices). The fronts are then reconstructed at any time as the zero level set of the $U$-interpolated function: since in a short-time evolution they are not supposed to move so far, for simplicity we have not used in the experiments the dynamic mesh algorithm proposed in [30], even though such an approach would allow us to strongly reduce the number of elements far from the fronts, at least when they are well separated.

\section{Experiments}

In the computer simulations we have done, the two models have exhibited similar performances. The stopping time has been chosen empirically on the basis of a number of experiments. In this section we discuss some of them, stressing more on the qualitative aspects than on the technicalities.

A first test was made to verify in practice the area conservation property of the models: the evolution of a single flower-shaped curve was considered and the area contained in it was computed at any time iteration. Even for an $\varepsilon$ of the order of $10^{-1}$ only, its shape rapidly converges to a disc of equivalent size, see Fig. 1. In Table 1 the initial and final values of 'mass' and 'area' are reported, respectively computed as

$$
m(n)=\int_{\Omega} U\left(t_{n}\right) \mathrm{d} x, \quad a(n)=\left|\Omega_{n}^{+}\right|-\left|\Omega_{n}^{-}\right|,
$$

with

$$
\Omega_{n}^{+}=\left\{x \in \Omega: U\left(t_{n}\right)(x)>0\right\}, \quad \Omega_{n}^{-}=\Omega-\Omega_{n}^{+} .
$$



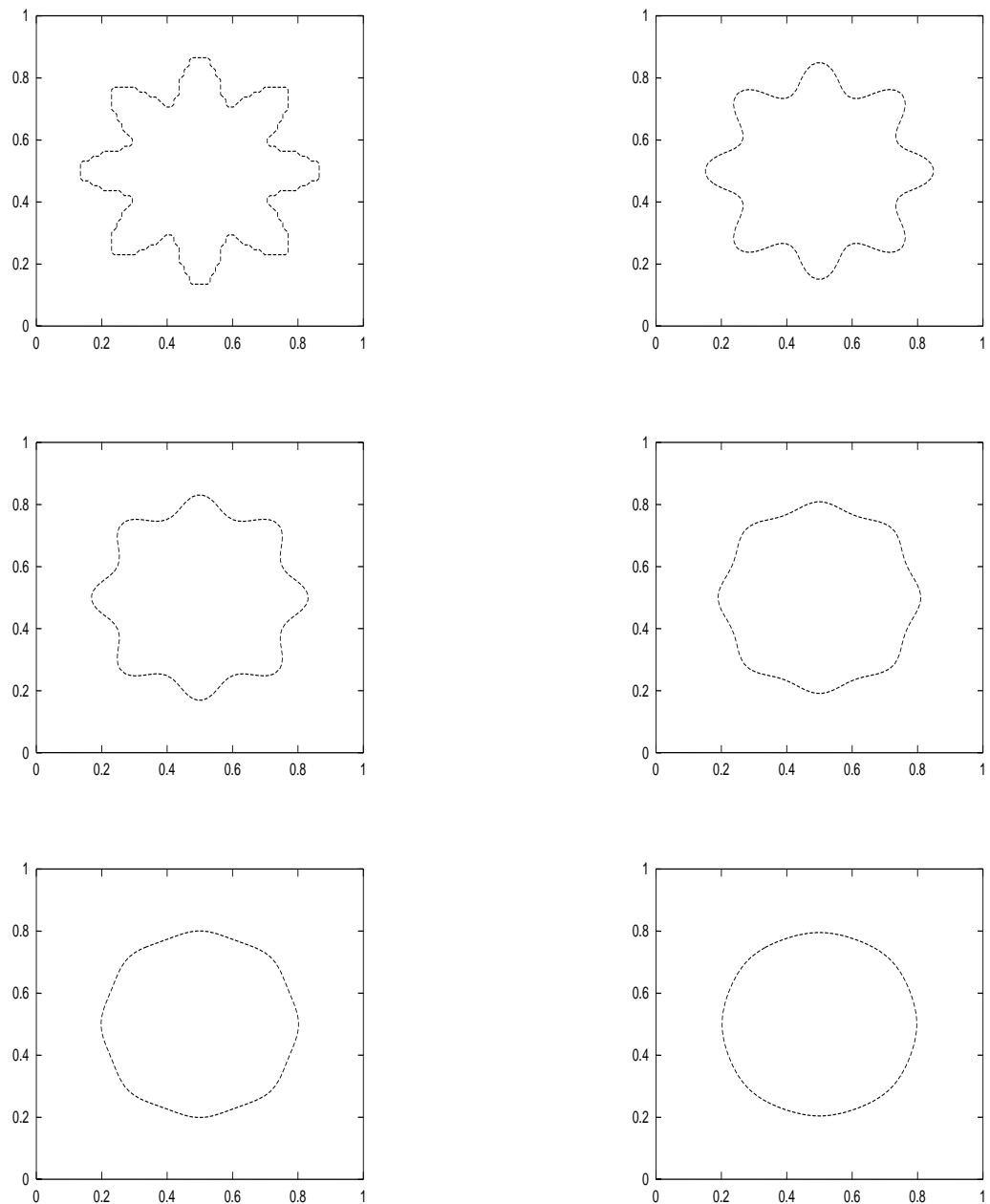

FIG. 1. Cahn-Hilliard evolution of a single curve, $h=1 / 63, \Delta t=10^{-5}, \varepsilon=10^{-1}$, time iterations $n=0$ (the initial front), $1,2,4,6$ and 10.

Since the initial datum $U(0)$ is a two-value vector (equal to -1 inside the curve and +1 outside), for $n=0$ the two quantities coincide. After ten iterations the variation is less than $5 \%$.

The effects of the coarsening property were studied on a long-time (Cahn-Hilliard) evolution of a more complex, even if still artificial, black and white image. In this case the initial set of curves can be directly chosen as a level set of the interpolated intensity function. Figure 2 shows that when the diffusion term in the equation is not negligible, different fronts can merge together as time increases. Further iterations would transform the shape into an annulus and, finally, into a disc. For a smaller $\varepsilon$, on the contrary, the three balls would vanish independently without merging.

For the same image, perturbed with some artificial noise, we experimented with the filtering effects of the algorithms. The Cahn-Hilliard approach (see Fig. 3) shows that small blobs due to 
TABLE 1

Values of mass and area for the curve evolution of Fig. 1

\begin{tabular}{lcc}
\hline Iteration & Mass & Area \\
\hline$n=0$ & 0.421517 & 0.421517 \\
$n=10$ & 0.403177 & 0.443689 \\
\hline
\end{tabular}
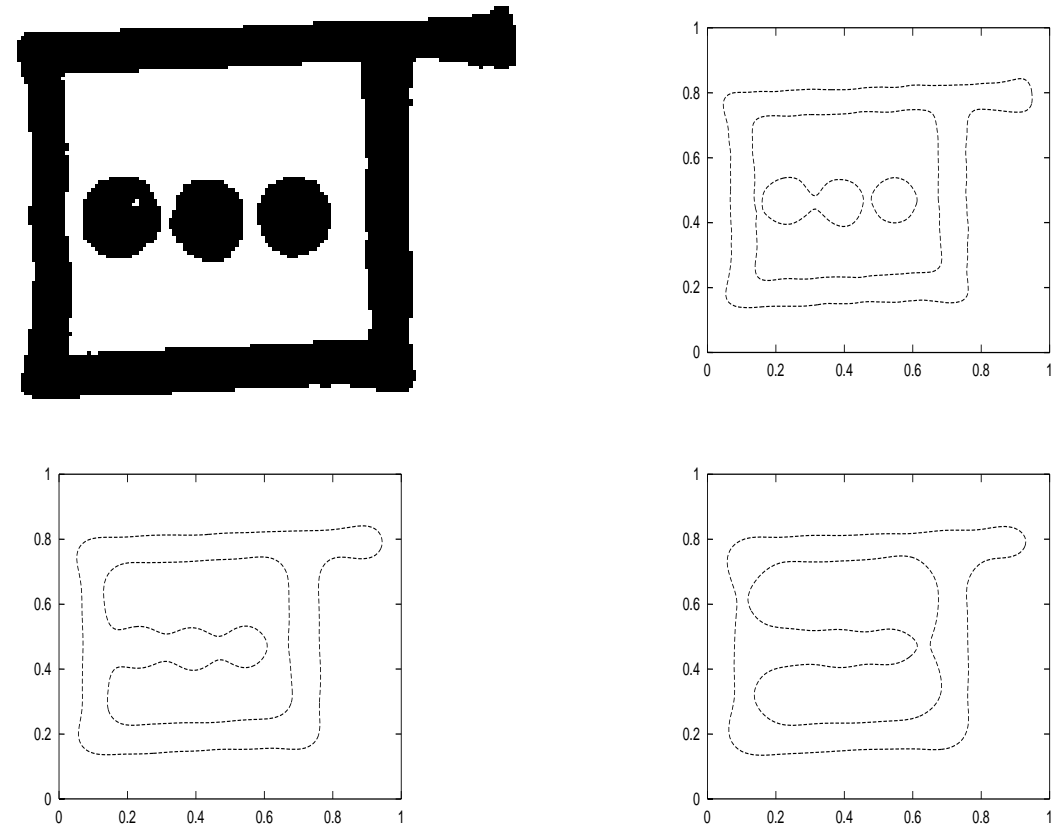

FIG. 2. Evolution of a black and white image for the Cahn-Hilliard flow: the original image and its evolving contours after 1,4 and 10 time iterations $\left(h=1 / 149, \Delta t=10^{-6}, \varepsilon=10^{-1}\right)$.

the noise disappear after a short time while the true contours are smoothed without shrinkage, in accordance with the theory: then the coarsening property acts like a denoising factor in the evolution.

We also tested the algorithms on real images: Fig. 4(a) shows a picture $(256 \times 256)$ of a cell nucleus taken by an electronic microscope. In that case, the straightforward use of the zero-crossings set $\Gamma_{*}$ given in (4.1) is not very helpful, since it produces too many curves and makes very hard the reconstruction of a function with such a zero level set to be used as initial datum for the evolution.

Then we adopted a slightly different strategy: the original image was pre-processed by a threshold method in order to concentrate the attention only on the region of main interest (the nucleus in this case, Fig. 4(b)), then the initial binary data for the evolution was created using in a suitable way the sign information on the Laplacian of the (smoothed) intensity function of the image ((c) and (d)). A short-time evolution with the nonlocal Allen-Cahn model shows in the 

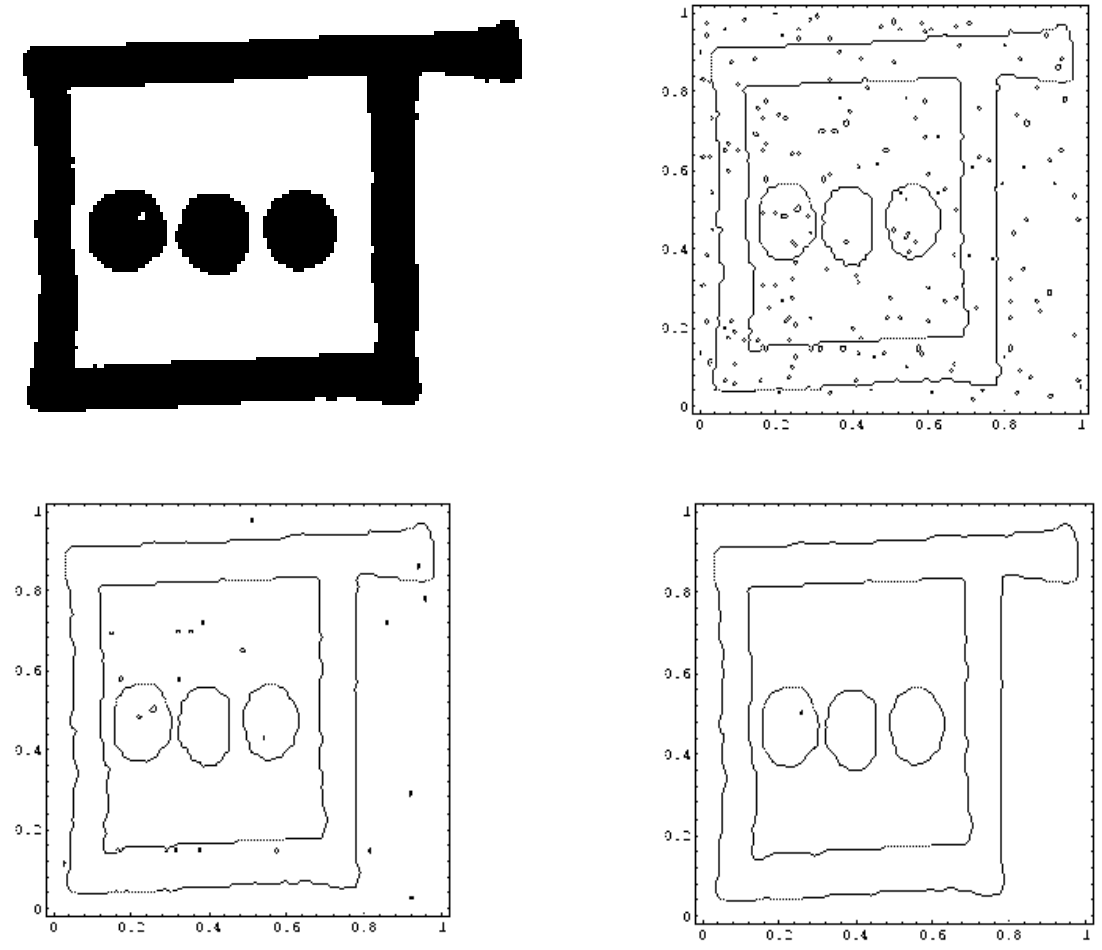

FIG. 3. The Cahn-Hilliard processing: the initial image, the initial set of curves (with noise), the zero level set after 4 and 10 iterations $\left(h=1 / 120, \Delta t=10^{-8}, \varepsilon=10^{-2}\right)$.

last two pictures how the smaller details of the image are quickly filtered while the contours are smoothed without affecting the size of the cell.

\section{REFERENCES}

1. Alikakos, N., Bates, P., \& Chen, X. Convergence of the Cahn-Hilliard equation to the HeleShaw model. Arch. Rational Mech. Anal. 128, (1994) 165-205.

2. ALLEN, S. M. \& CAHN, J. W. A microscopic theory for antiphase boundary motion and its application to antiphase domain coarsening. Acta Met. 27, (1979) 1085-1095.

3. Alvarez, L., Guichard, F., Lions, P. L., \& Morel, J. M. Axioms and fundamental equations of image processing. Arch. Rational Mech. Anal. 123, (1993) 199-257.

4. Blowey, J. F. \& ElliotT, C. M. The Cahn-Hilliard gradient theory for phase separation with nonsmooth free energy. Part II: Numerical analysis. Euro. J. Appl. Math. 3, (1992) 147-179.

5. BRONSARD, L. \& KoHN, R. V. Motion by mean curvature as a singular limit of GinzburgLandau dynamics. J. Diff. Eqs. 90, (1991) 211-237.

6. BRONSARD, L. \& STOTH, B. Volume-preserving mean curvature flow as a limit of a nonlocal GinzburgLandau equation. SIAM J. Math. Anal. 28, (1997) 769-807.

7. Capuzzo Dolcetta, I. \& Ferretti, R. Optimal stopping time formulation of adaptive image filtering. Appl. Math. and Optimiz 43, (2001) 245-258. 

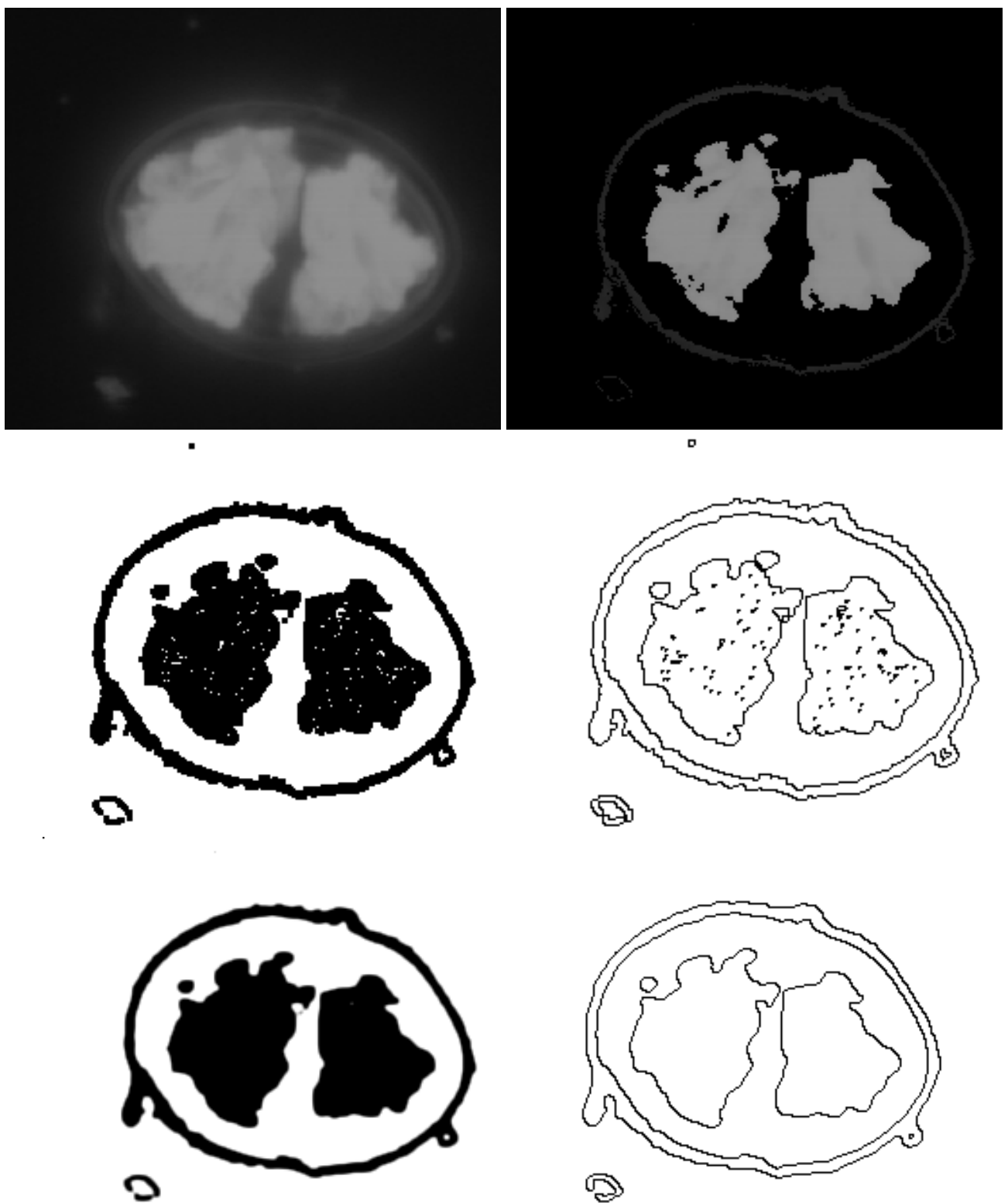

FIG. 4. The nonlocal Allen-Cahn processing: the initial image (a) and its pre-processing (b); the initial data (c) and its zero level set (d); the final image after ten iterations (e) and its zero level set (f) $\left(h=1 / 255, \Delta t=3 \times 10^{-6}, \varepsilon=3 \times 10^{-3}\right)$. 
8. Capuzzo Dolcetta, I., Finzi Vita, S., \& March, R. A phase transition approach to planar curve evolution without shrinkage. Proc. of the MTNS98 Conference. Padova (1998) pp. 451-454.

9. Caselles, V., Catté, F., Coll, T., \& Dibos, F. A geometric model for active contours in image processing. Numer. Math. 66, (1993) 1-31.

10. Chen, X. The Hele-Shaw problem and area-preserving curve-shortening motions. Arch. Rational Mech. Anal. 123, (1993) 117-151.

11. Chen, X. Global asymptotic limit of solutions of the Cahn-Hilliard equations. J. Diff. Geom. 47, (1996) 262-311.

12. Chen, Y.-G., Giga, Y., \& Goto, S. Uniqueness and existence of viscosity solutions of generalized mean curvature flow equations. J. Diff. Geom. 33, (1991) 749-786.

13. De Mottoni, P. \& Schatzman, M. Evolution géométrique d'interfaces. C.R. Acad. Sci. Paris Sér. I Math. 309, (1989) 453-458.

14. Elliott, C. M., French, D. A., \& Milner, F. A. A second order splitting method for the CahnHilliard equation. Num. Math. 54, (1989) 575-590.

15. Evans, L. C., SONER, H. M., \& Souganidis, P. E. Phase transitions and generalized motion by mean curvature. Comm. Pure Appl. Math. 45, (1992) 1097-1123.

16. Evans, L. C. \& SPRUCK, J. Motion of level sets by mean curvature. J. Diff. Geom. 33, (1991) 635-681.

17. FINZI VITA, S. A Cahn-Hilliard equation with non homogeneous mobility and its application to image processing. Proc. of the ENUMATH 2001 Conference, submitted. Ischia (2001).

18. GAGE, M. Curve shortening makes convex curves circular. Invent. Math. 76, (1984) 357-364.

19. GAGE, M. On an area-preserving evolution equation for plane curves. Contemp. Math. 51, (1986) 51-62.

20. Gage, M. \& Hamilton, R. S. The heat equation shrinking convex plane curves. J. Diff. Geom. 23, (1986) 69-96.

21. Grayson, M. The heat equation shrinks embedded plane curves to round points. J. Diff. Geom. 26, (1987) 285-314.

22. Grayson, M. Shortening embedded curves. Annals of Mathematics 129, (1989) 71-111.

23. Kichenassamy, S., Kumar, A., Olver, P., Tannenbaum, A., \& Yezzi, Jr. A. Conformal curvature flows: from phase transitions to active vision. Arch. Rational Mech. Anal. 134, (1996) 275-301.

24. KimiA, B. B. \& SiddiQI, K. Geometric heat equation and nonlinear diffusion of shapes and images. Computer Vision and Image Understanding 64, (1996) 305-322.

25. Kimia, B. B., Tannenbaum, A., \& Zucker, S. W. Shapes, shocks, and deformations, I: the components of shape and the reaction-diffusion space. Int. J. Comput. Vision 15, (1995) 189-224.

26. Malladi, R., Sethian, J. A., \& Vemuri, B. C. Shape modeling with front propagation: a level set approach. IEEE Trans. PAMI 17, (1995) 158-175.

27. MARCH, R. \& DOZIO, M. A variational method for the recovery of smooth boundaries. Image and Vision Computing Journal 15, (1997) 705-712.

28. Marr, D. \& Hildreth, E. Theory of edge detection. Proc. Roy. Soc. Lond. Ser. B 207, (1980) 187-217.

29. Mokhatarian, F. \& MaCKWORTH, A. A theory of multi-scale, curvature based shape representation for planar curves. IEEE Trans. PAMI 14, (1992) 789-805.

30. Nochetto, R. H., Paolini, M., \& Verdi, C. A dynamic mesh algorithm for curvature dependent evolving interfaces. J. Comput. Phys. 123, (1996) 296-310.

31. Nochetto, R. H. \& Verdi, C. Approximating curvature driven interfaces with applications to shape recovery. Curvature flows and related topics (Levico, 1994), Gakuto Internat. Ser. Math. Sci. Appl. 5, (1995) 159-177.

32. Novick-Cohen, A. On the viscous Cahn-Hilliard equation. In: BALL, J. (ed), Material Instabilities in Continuum Mechanics and Related Mathematical Problems. pp. 329-342. Clarendon, Oxford (1988).

33. Osher, S. J. \& Sethian, J. A. Fronts propagation with curvature dependent speed: algorithms based on Hamilton-Jacobi formulations. J. Comp. Phys. 79, (1988) 12-49.

34. Pego, R. L. Front migration in the nonlinear Cahn-Hilliard equation. Proc. Roy. Soc. Lond. Ser. A 422, (1989) 261-278. 
35. Rubinstein, J. \& Sternberg, P. Nonlocal reaction-diffusion equations and nucleation. IMA J. of Appl. Math. 48, (1992) 249-264.

36. Rubinstein, J., Sternberg, P., \& Keller, J. B. Fast reaction, slow diffusion, and curve shortening. SIAM J. Appl. Math. 49, (1989) 116-133.

37. Sapiro, G. \& Tannenbaum, A. Affine invariant scale-space. Int. J. Comput. Vision 11, (1993) 25-44.

38. Sapiro, G. \& TANnenbaum, A. Area and length preserving geometric invariant scale-spaces. IEEE Trans. PAMI 17, (1995) 67-72.

39. Shah, J. Uses of elliptic approximations in computer vision. In: Serapioni, R. \& Tomarelli, F. (eds), Variational Methods for Discontinuous Structures. pp. 19-34. Birkhauser, Boston (1996).

40. Torre, V. \& Poggio, T. On edge detection. IEEE Trans. PAMI 8, (1986) 147-163. 